\title{
Efficacy of Vibegron and Mirabegron for Overactive Bladder: A Systematic Literature Review and Indirect Treatment Comparison
}

\author{
Michael J. Kennelly (D) · Thomas Rhodes • Cynthia J. Girman • \\ Elizabeth Thomas · Denise Shortino - Paul N. Mudd Jr.
}

Received: July 6, 2021 / Accepted: August 24, 2021 / Published online: September 18, 2021

(C) The Author(s) 2021

\begin{abstract}
Background: In the absence of head-to-head trials, we performed an indirect treatment comparison of the $\beta_{3}$-adrenergic agonists vibegron and mirabegron in the treatment of overactive bladder (OAB).

Methods: PubMed, Embase, and Cochrane Library were searched for articles related to phase 3 , double-blind, controlled trials of vibegron $75 \mathrm{mg}$ and mirabegron $25 / 50 \mathrm{mg}$ in patients with OAB. Efficacy outcomes included change from baseline at weeks 4,12 , and 52 in mean daily number of total urinary incontinence episodes and micturitions and mean volume voided/micturition. Effect size was computed as placebo-subtracted change from
\end{abstract}

Paul Mudd and Denise Shortino were employees of Urovant Sciences at the time the work was conducted.

Supplementary Information The online version contains supplementary material available at https:// doi.org/10.1007/s12325-021-01902-8.

M. J. Kennelly ( $₫)$

Department of Urology, Obstetrics and Gynecology,

Carolinas Medical Center, 2001 Vail Ave, Suite 360,

Charlotte, NC 28207, USA

e-mail: Michael.Kennelly@atriumhealth.org

T. Rhodes · C. J. Girman

CERobs Consulting, LLC, Chapel Hill, NC, USA

E. Thomas - D. Shortino - P. N. Mudd Jr.

Urovant Sciences, Irvine CA USA baseline (weeks 4, 12) or active control (tolterodine)-subtracted change from baseline (week 52) for each treatment group. Adverse events (AEs) are presented descriptively.

Results: After removal of duplicates, 49 records were identified, and after screening 9 met inclusion criteria for analysis. Vibegron showed significantly greater reduction in mean daily number of total incontinence episodes than mirabegron $25 \mathrm{mg}$ at week 4, mirabegron $50 \mathrm{mg}$ (weeks 4, 52), and tolterodine (weeks 4, 12) $(P<0.05$, each) and significantly greater improvement in volume voided versus mirabegron $25 \mathrm{mg}$ (week 12), mirabegron $50 \mathrm{mg}$ (weeks 12, 52), and tolterodine (week 4) $(P<0.05$, each). Confidence intervals of point estimates overlapped zero for all other comparisons of vibegron and mirabegron ( 25 or $50 \mathrm{mg}$ ) or tolterodine, indicating no significant differences between treatments for these time/endpoints. Urinary tract infection, hypertension, and dry mouth were the most commonly occurring AEs for vibegron, mirabegron, and tolterodine, respectively, in the short-term trials; hypertension was the most commonly occurring AE with all three treatments in the long-term trials.

Conclusions: Vibegron was associated with significant improvement in total incontinence episodes versus mirabegron at 4 and 52 weeks and volume voided at 12 and 52 weeks. Improvement in micturitions was similar between vibegron and mirabegron or 
tolterodine. Incidence of AEs was generally comparable between vibegron and mirabegron.

Keywords: Adrenergic agonists; Urinary bladder; Overactive; Urinary incontinence

\section{Key Summary Points}

Why carry out this study?

Oral pharmacologic treatments for overactive bladder include anticholinergics and $\beta_{3}$-adrenergic receptor agonists; however, anticholinergics are associated with bothersome side effects and low persistence that may be improved with $\beta_{3}$ adrenergic agonists

In the absence of head-to-head trials for the $\beta_{3}$-adrenergic receptor agonists vibegron and mirabegron as monotherapy, we performed a systematic literature review and indirect treatment comparison of efficacy outcomes from nine clinical trials in patients with overactive bladder

\section{What was learned from study?}

Vibegron was associated with significant improvements in total incontinence episodes compared with mirabegron at weeks 4 and 52 and in volume voided compared with mirabegron at weeks 12 and 52; no significant differences in micturitions were seen between vibegron and mirabegron or tolterodine (active control)

Rates of adverse events were generally comparable between vibegron and mirabegron

Further studies are needed to evaluate whether improved long-term efficacy associated with vibegron in clinical trials translates into improved treatment persistence in real-world settings

\section{INTRODUCTION}

Bothersome symptoms of overactive bladder $(\mathrm{OAB})$ affect an estimated 30 million adults in the USA [1]. Clinical guidelines recommend behavioral therapy with or without pharmacologic management as first-line treatment for $\mathrm{OAB}$ and oral anticholinergics or $\beta_{3}$-adrenergic agonists as second-line treatment [2]. However, anticholinergics are associated with adverse effects such as dry mouth, blurred vision, and constipation [3], as well as increased incidence of falls [4] and risk of dementia [5]. Treatment persistence at 12 months with anticholinergics for $\mathrm{OAB}$ is poor $(\sim 22 \%)$ [6], and about $46 \%$ of patients cite lack of efficacy as a reason for discontinuing [7].

The introduction of $\beta_{3}$-adrenergic agonists has improved $\mathrm{OAB}$ management by minimizing anticholinergic-related adverse effects [8], leading to improved treatment persistence [6] and effectiveness. However, the onset of action of mirabegron-a $\beta_{3}$-adrenergic agonist approved in the USA for the treatment of $\mathrm{OAB}$ - may take up to 8 weeks at the recommended $25-\mathrm{mg}$ starting dose or 4 weeks at a 50-mg dose [9], suggesting dose escalation from 25 to $50 \mathrm{mg}$ may be necessary. Furthermore, the maximum dose of mirabegron is $25 \mathrm{mg}$ for patients with severe renal or moderate hepatic impairment [10]. Mirabegron and solifenacin combination therapy can improve long-term efficacy but is associated with increased adverse events (AEs) [11]. Monotherapy with a $\beta_{3}$-adrenergic agonist may be preferred in older patients, those with high anticholinergic burden, and older adults with multiple comorbidities. Vibegron is a selective $\beta_{3}$-adrenergic agonist that was approved by the US Food and Drug Administration at a once-daily dose of $75 \mathrm{mg}$ for the treatment of OAB $[12,13]$. Dosage adjustments are not recommended for patients with renal or mild to moderate hepatic impairment [14]. Although both are $\beta_{3}$-adrenergic agonists, vibegron and mirabegron display differing selectivity and maximum response at $\beta_{3}$-adrenergic receptors [15].

Individual, phase 3 clinical trials have compared vibegron $75 \mathrm{mg}$ or mirabegron 25 or 
$50 \mathrm{mg}$ with placebo and have included anticholinergics as active controls; however, no trial has assessed the comparative efficacy of vibegron versus mirabegron. In such situations, indirect treatment comparisons can be useful to assess differential efficacy between active treatment groups and are commonly used by governing and health technology assessment bodies in the absence of head-to-head trials. We performed a systematic literature review and indirect treatment comparison of efficacy measures for vibegron $75 \mathrm{mg}$ and mirabegron 25 or $50 \mathrm{mg}$ for the treatment of OAB.

\section{METHODS}

\section{Search and Selection Criteria}

This systematic literature review was conducted in accordance with Preferred Reporting Items for Systematic Review and Meta-Analyses (PRISMA) reporting guidelines. PubMed, Embase, and the Cochrane Library were searched for English language articles using terms related to phase 3 , double-blind, controlled trials of vibegron and mirabegron in patients with OAB (Supplementary Table 1). Searches were performed in August 2020. Articles were excluded if they were reviews, pooled analyses, or studies in populations other than $\mathrm{OAB}$ (e.g., benign prostatic hyperplasia, neurogenic bladder). A manual search of primary publications and references was performed to identify relevant articles not captured during the initial search. Previously unpublished data for two phase 3 studies of vibegron were provided by Urovant Sciences in the form of clinical study reports; data for the trials have since been published [12, 13].

Article screening was independently conducted by two experienced reviewers who were not involved in manuscript development; discrepancies were resolved by a third reviewer (TR). Titles and abstracts were screened, and if the study reported the outcome of interest and met inclusion criteria, the study was included for full-text review. Studies were included if they were a phase 3 , randomized, double-blind, placebo- or tolterodine-controlled trial of vibegron $75 \mathrm{mg}$, mirabegron $25 \mathrm{mg}$, or mirabegron $50 \mathrm{mg}$ for the treatment of patients at least 18 years old with OAB. Studies that included vibegron $50 \mathrm{mg}$ were omitted as the 50-mg dose is not approved in the USA. Studies were required to have at least 3 months' (12 weeks) exposure to study drug.

\section{Data Extraction and Risk of Bias}

One reviewer extracted information on source, study design, population, analysis, interventions, outcome assessments, and results using a standardized extraction form (Supplementary Table 2); a second reviewer verified extracted data against the original publications. Disagreements were resolved by consensus. When more than one publication existed for a study, reports were grouped, and the most recent publication with the most complete data or longest follow-up period was included. Any discrepancies between published versions were recorded.

Two reviewers independently evaluated risk of bias (graded as low, high, or unclear) and quality of the body of evidence (graded as high, moderate, low, or very low) using the Cochrane Risk of Bias Tool [16]. Disagreements were settled by consensus or arbitrated by a third reviewer (TR). Funnel plot asymmetry was examined, and plausible explanations for asymmetry were considered. The "trim and fill" method [17] was used to assess publication bias and estimate the effect corrected for by the bias.

\section{Outcomes and Data Synthesis}

Efficacy outcomes of interest included change from baseline at weeks 4,12 , and 52 in mean daily number of total urinary incontinence episodes, mean daily number of micturitions, and mean volume voided per micturition (in milliliters). Outcomes were based on a 3-day diary for mirabegron (except for a 7-day diary in Herschorn et al. [18]) and 7-day diary for vibegron, which have been shown to give comparable results [19]. Daily total urinary incontinence episodes were defined as the sum of all incontinence episodes in a 24 -h period, 
averaged across the relevant number of diary days. The definition of urgency and urge urinary incontinence differed among trials and therefore could not be assessed. Forest plots with 95\% confidence intervals (CIs) were generated for each outcome measure and time point. For continuous outcomes, mean difference in scores or difference in mean change scores with 95\% CIs were derived from summary statistics for each group (i.e., mean or mean change and SD and sample sizes).

Demographics were analyzed in the safety analysis sets (all patients who received at least one dose of study medication) unless otherwise indicated. Micturitions and volume voided were analyzed in the full analysis sets (all patients in the safety analysis set with at least one baseline and postbaseline assessment), and total incontinence episodes were analyzed in the full analysis sets for incontinence (all patients in the full analysis set with at least one incontinence episode at baseline). For weeks 4 and 12, effect size was computed as placebo-subtracted mean change from baseline for each treatment group. Differences between vibegron and mirabegron 25 or $50 \mathrm{mg}$ were computed using the placebosubtracted mean changes from baseline. Because no placebo group was included in the 52 -week vibegron or mirabegron trials, tolterodine $4 \mathrm{mg}$ extended release (active control) was used to compute tolterodine-subtracted change from baseline. For total incontinence episodes and micturitions, negative values indicate improvement (i.e., reduction) from baseline; for volume voided per micturition, positive values reflect improvement. Safety was assessed through $\mathrm{AE}$ reporting and is presented descriptively.

Heterogeneity was assessed using 95\% prediction intervals; variance of heterogeneity $\left(I^{2}\right)$ was calculated for reference. The $I^{2}$ statistic measures the inconsistency of study results by describing the percentage of variation across studies that is due to heterogeneity rather than chance. If $I^{2}$ was less than $50 \%$, a random effects model was used to create a summary estimate. If $I^{2}$ was $50 \%$ or more, no summary estimate was produced, and data from individual trials were described. The indirect treatment comparison was conducted using previously described methods [20]. The Bucher method assumes that the trials indirectly compared are comparable with respect to study population, study design, and outcomes; that the relative treatment effect is the same across the trials; and that pairwise comparisons are independent. It also assumes the $\log$ of the effect size measured for drug A versus drug $B$ equals the difference of the $\log$ effect size measures, which is supported if assumptions hold. Egger's test is a linear regression of the intervention effect estimates on their standard errors weighted by their inverse variance and was used to identify publication bias in the meta-analyses by assessing asymmetry in a funnel plot. Statistical analyses were performed using Stata, release 16.1 (College Station, TX; StataCorp LLC).

\section{Compliance with Ethics Guidelines}

These analyses are based on previously published studies in which ethics approval and informed consent were obtained. As a result, institutional review board approval and written informed consent were not needed.

\section{RESULTS}

\section{Studies}

A total of 16 records were identified through the initial database search, and 57 were identified by hand searches (Fig. 1). After removal of duplicates, 49 records remained and were screened for inclusion; 34 records did not meet inclusion criteria, resulting in 15 full-text articles being assessed for eligibility. After further evaluation, seven were identified and included in the meta-analyses [18, 21-26], in addition to the vibegron phase 3 EMPOWUR [12] and EMPOWUR extension [13] trials.

The nine included trials had similar enrollment criteria, which included patients at least 18 years old with $\mathrm{OAB}$ for at least 3 months (at least 20 years old with $\mathrm{OAB}$ for at least 24 weeks in Yamaguchi et al. [25]) with or without urinary incontinence. Patients were required to have at least eight micturitions per day and at 


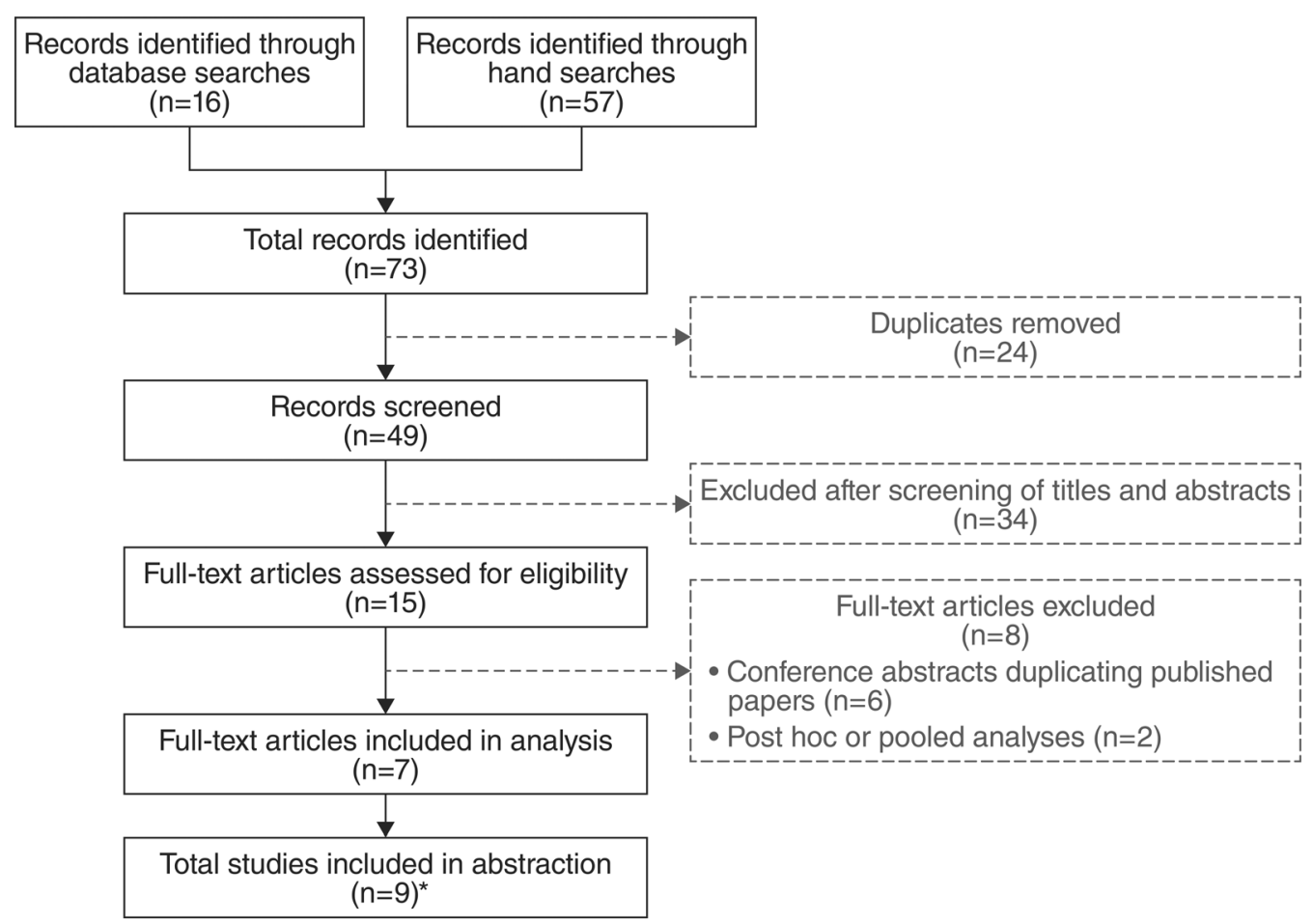

Fig. 1 PRISMA flow diagram. *Includes two studies that were unpublished at the time in which data were provided by Urovant Sciences

least one $[18,25,26]$ or at least three $[12,21-24]$ urgency episodes. Urgency in the mirabegron trials was typically defined as grade 3 (severe urgency) or 4 (urge incontinence) on the Patient Perception of Intensity of Urgency Scale; in the vibegron trials, urgency was defined as the need to urinate immediately. The trials comprised more than 14,000 patients with OAB; outcomes from more than 10,000 patients were included in the analyses (vibegron, $n=526$; mirabegron $25 \mathrm{mg}, n=855$; mirabegron $50 \mathrm{mg}, n=3316$; tolterodine, $n=2425$; placebo, $n=3020$; Table 1 ). Of the studies, seven were 12 -week studies, one was 52 weeks, and one was an extension study of the 12-week trial with up to 52 weeks of exposure. Common comparators for vibegron and mirabegron were placebo and tolterodine (Supplementary Fig. 1).

Baseline demographics were well balanced within trials and were generally similar across trials (Table 1). Mean patient age across all trials ranged from 54.5 to 61.1 years. Most trial participants were women (range 67-85\%), and with the exception of trials conducted in Asia, most participants were white (range 76.6-99.1\%). At baseline, mean daily micturitions ranged from 10.8 to 12.6 , mean daily incontinence episodes ranged from 1.9 to 4.2 , and volume voided/micturition ranged from 145.9 to $165.2 \mathrm{~mL}$ (Supplementary Table 3). The percentage of patients with $\mathrm{OAB}$ and urinary incontinence ranged from $58.0 \%$ to $100 \%$.

\section{Efficacy Outcomes}

\section{Mean Daily Total Incontinence Episodes}

At weeks 4 and 12, all four active treatments had significantly greater reduction from baseline than placebo in total incontinence episodes. At week 52, vibegron had significantly greater reduction than tolterodine (Supplementary Fig. 2). At week 4, vibegron showed significantly greater reduction in mean daily number of total incontinence episodes compared with mirabegron (both doses) and tolterodine $(P<0.05$, each; Fig. 2 ; Supplementary Table 4). At week 12, vibegron showed significantly greater reduction in total incontinence 


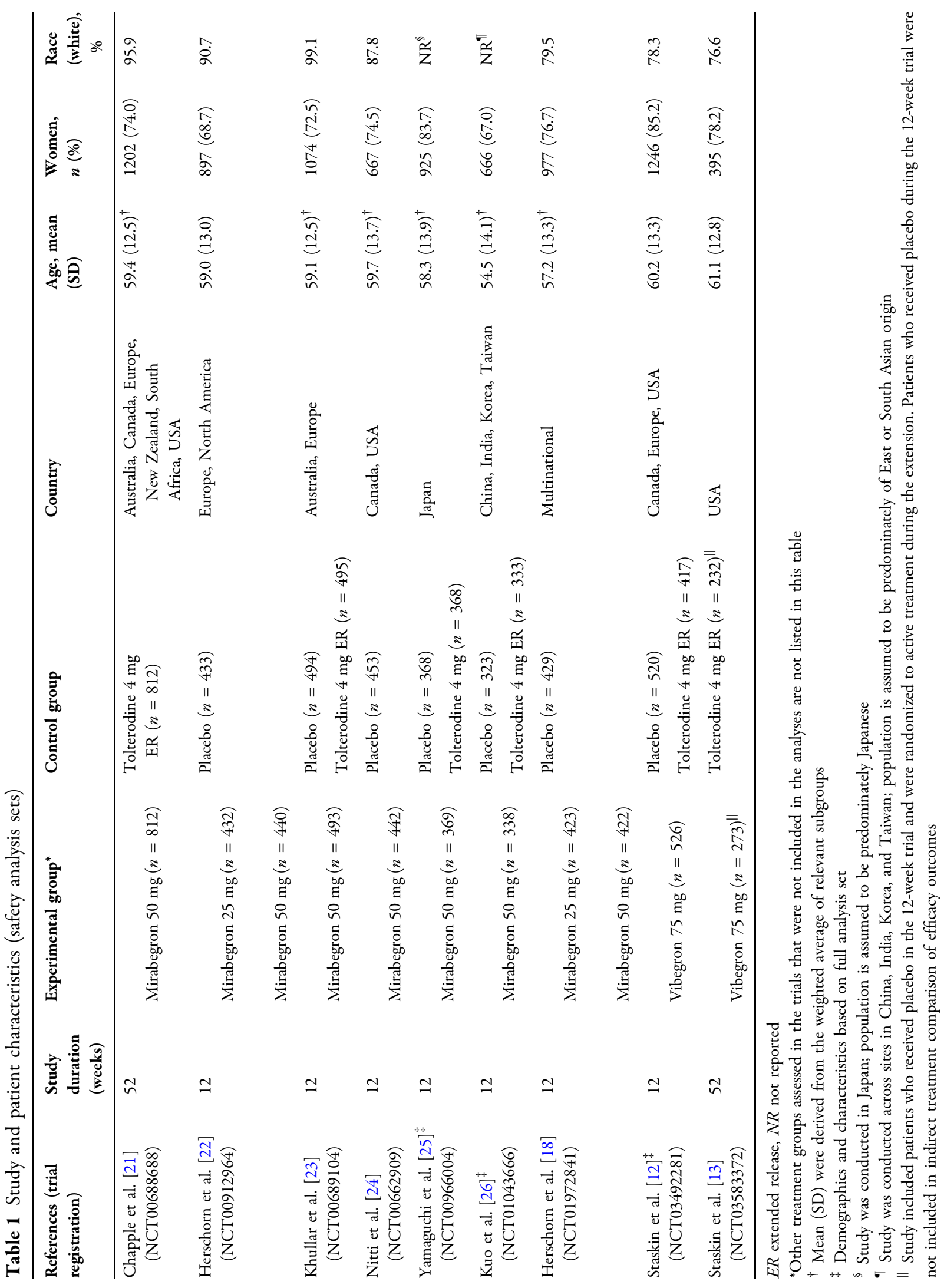




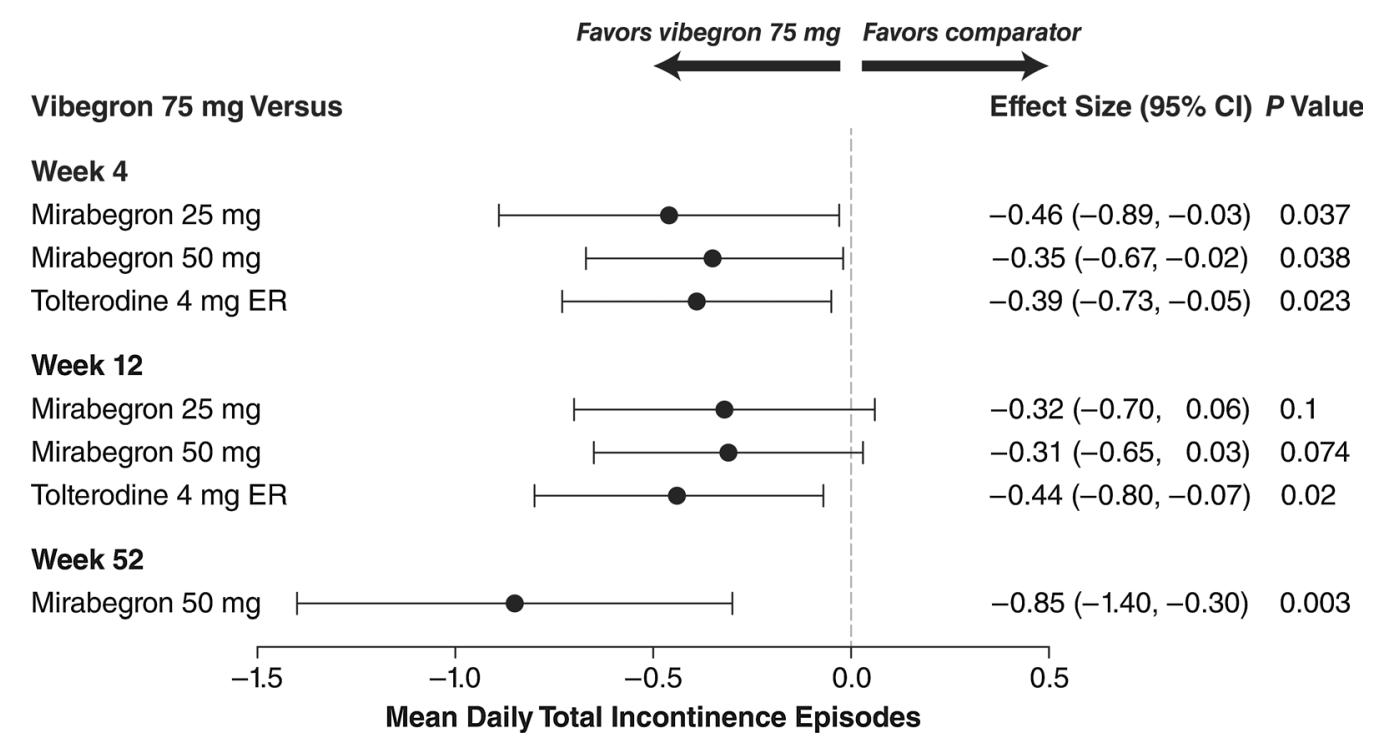

Fig. 2 Indirect treatment comparison of change from baseline in mean daily total incontinence episodes (full analysis set for incontinence) based on a 3- or 7-day diary (for mirabegron and vibegron, respectively). Effect sizes at weeks 4 and 12 were calculated using placebo-subtracted

episodes than tolterodine $(P<0.05)$. At week 52, vibegron showed significantly greater reduction in total incontinence episodes versus mirabegron $50 \mathrm{mg}(P<0.01)$.

\section{Mean Daily Micturitions}

At week 4, all active treatment groups except mirabegron $25 \mathrm{mg}$ had significantly greater reduction from baseline than placebo in micturitions; all active treatments had significantly greater reduction versus placebo at week 12 . Neither vibegron nor mirabegron was significantly different than tolterodine at week 52 (Supplementary Fig. 3). Confidence intervals for point estimates overlapped zero for all seven comparisons between vibegron and mirabegron 25 or $50 \mathrm{mg}$ and between vibegron and tolterodine, indicating no statistically significant differences between treatments across all time points (Fig. 3; Supplementary Table 4).

\section{Volume Voided Per Micturition}

At week 4, only vibegron and mirabegron $50 \mathrm{mg}$ had significantly greater improvement from baseline than placebo in volume voided; all active treatments had significantly greater changes from baseline; effect size at week 52 was calculated using tolterodine-subtracted changes from baseline. Negative effect sizes favor vibegron; positive effect sizes favor comparators. ER extended release

improvement versus placebo at week 12; and only vibegron had significantly greater improvement than tolterodine at week 52 (Supplementary Fig. 4). Volume voided was not reported for mirabegron $25 \mathrm{mg}$ at 4 or 52 weeks or for tolterodine at 52 weeks. At week 4, vibegron showed significantly greater improvement in volume voided versus tolterodine $(P<0.05$; Fig. 4; Supplementary Table 4). At week 12, vibegron showed significantly greater improvement in volume voided versus both doses of mirabegron (25 mg: $P<0.01 ; 50 \mathrm{mg}: P<0.05)$. At week 52, vibegron showed significantly greater improvement versus mirabegron $50 \mathrm{mg}$ $(P<0.05)$.

\section{Heterogeneity}

$I^{2}$ was less than $50 \%$ within treatment groups and outcomes, suggesting minimal heterogeneity within treatment groups, with the exception of week 12 values for volume voided per micturition with tolterodine (Cochran's Q $\left.P=0.03 ; I^{2}=67 \%\right)$. 


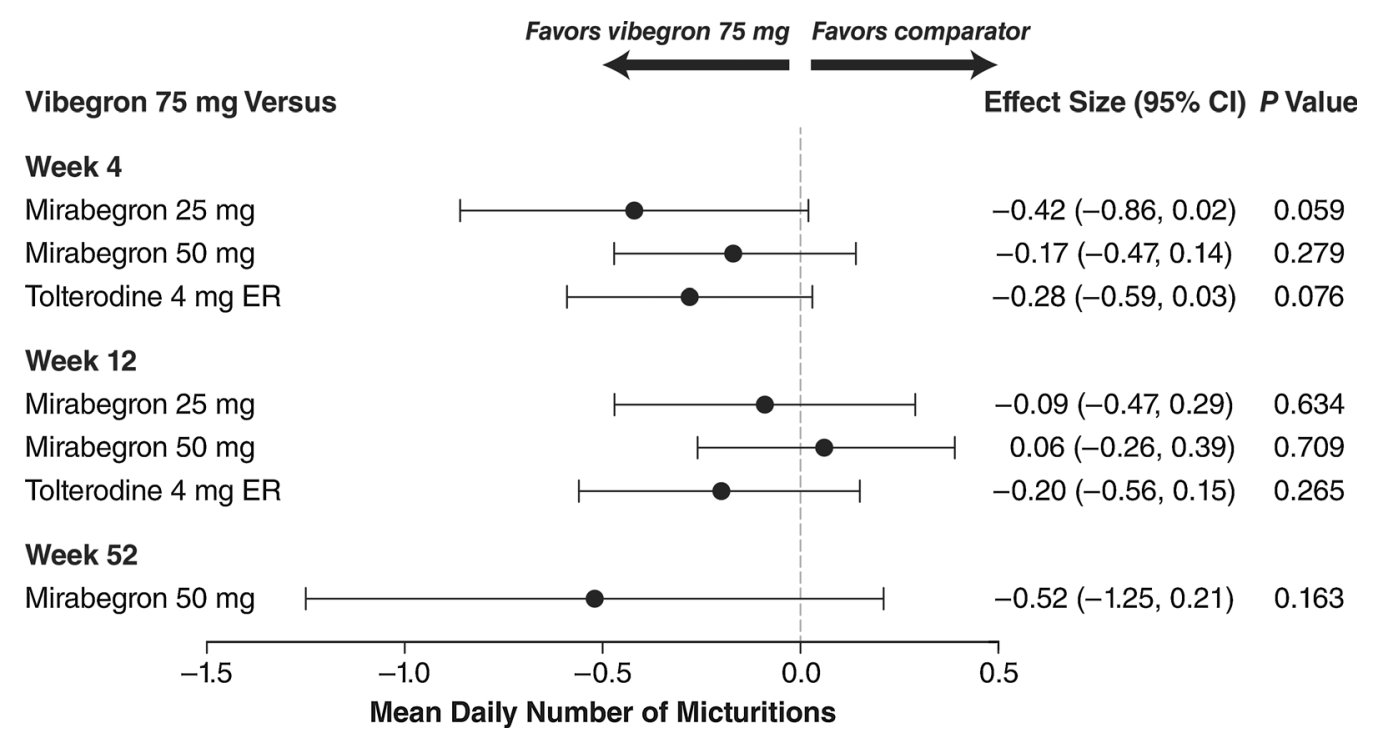

Fig. 3 Indirect treatment comparison of change from baseline in mean daily micturitions (full analysis set) based on a 3- or 7-day diary (for mirabegron and vibegron, respectively). Effect sizes at weeks 4 and 12 were calculated

\section{Risk of Bias}

Egger's regression test did not detect significant publication bias. Risk of bias was assessed to be low across all studies and criteria, and quality level was assessed as high for all studies.

\section{Safety}

Only the publications by Chapple et al. [21], Herschorn et al. [22], Khullar et al. [23], Nitti et al. [24], Yamaguchi et al. [25], Kuo et al. [26], and Staskin et al. [13] reported the incidence of AEs that occurred in at least $2 \%$ of any treatment group. Staskin et al. [12] reported AEs with an incidence of at least $2 \%$ in the vibegron group and greater than for placebo. Across all short-term trials reporting such data, the most frequently occurring $\mathrm{AE}$ with mirabegron 25 or $50 \mathrm{mg}$ was hypertension (mostly $0.3-11.3 \%$ of patients), with vibegron was urinary tract infection (UTI; 5\%), and with tolterodine was dry mouth (6.5-13.3\%; Supplementary Table 5). In the short-term trials, additional AEs reported at an incidence of at least $5 \%$ were nasopharyngitis with mirabegron $50 \mathrm{mg}$ and using placebo-subtracted changes from baseline; effect size at week 52 was calculated using tolterodine-subtracted changes from baseline. Negative effect sizes favor vibegron; positive effect sizes favor comparators. $E R$ extended release

hypertension and UTI with tolterodine $[12,22-26]$. Across the long-term trials, the most frequently occurring $\mathrm{AE}$ with mirabegron $50 \mathrm{mg}$, vibegron, and tolterodine was hypertension $(9.2 \%, 8.8 \%$, and $8.6-9.6 \%$, respectively; Supplementary Table 6). Additional AEs reported at an incidence of at least 5\% were UTI with mirabegron $50 \mathrm{mg}$; UTI and headache with vibegron; and UTI, dry mouth, and nasopharyngitis with tolterodine $[13,21]$.

\section{DISCUSSION}

This indirect treatment comparison assessed the efficacy of vibegron and mirabegron for the treatment of $\mathrm{OAB}$. In the clinical trials, vibegron and both doses of mirabegron showed significant improvement versus placebo in all assessed efficacy measures at week 12 . In this indirect treatment comparison, vibegron showed significantly greater improvement in total urinary incontinence episodes versus mirabegron 25 and $50 \mathrm{mg}$ and tolterodine at week 4 , versus tolterodine at week 12 , and versus mirabegron $50 \mathrm{mg}$ at week 52. Vibegron also showed significantly greater improvement in volume 


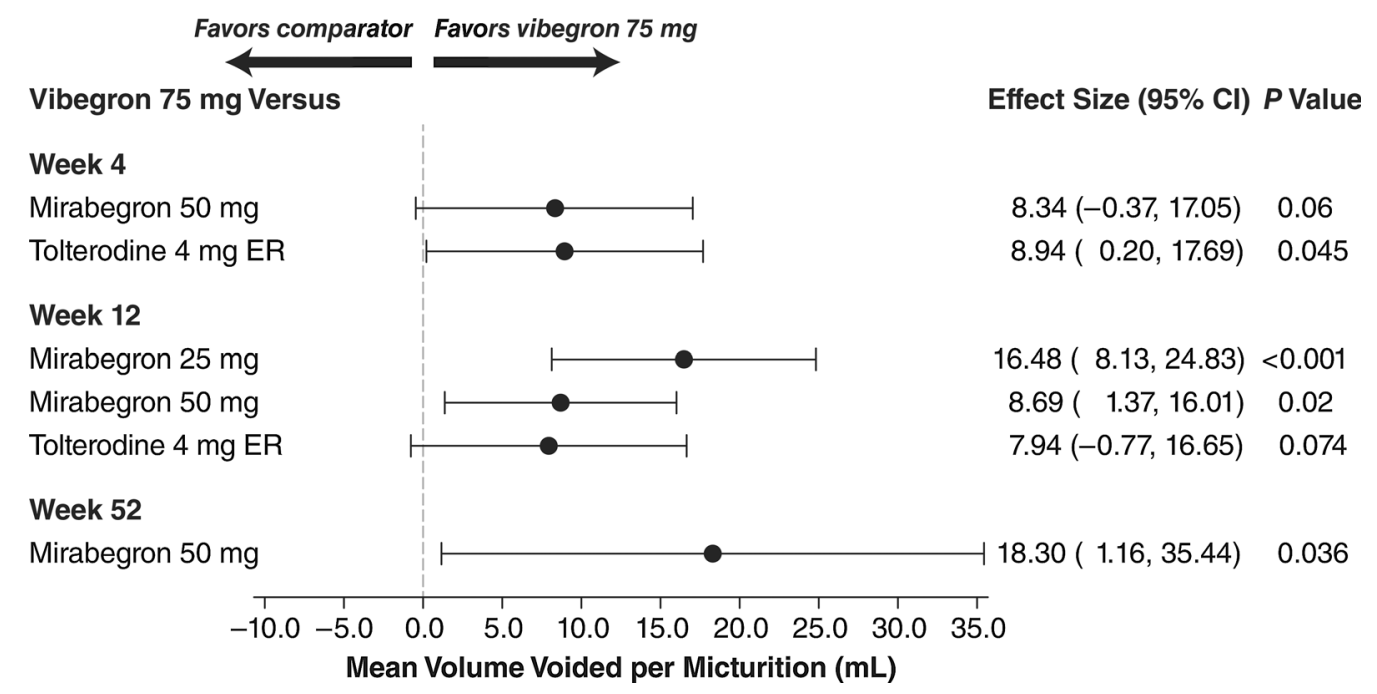

Fig. 4 Indirect treatment comparison of change from baseline in mean volume voided per micturition (milliliters; full analysis set) based on a 3- or 7-day diary (for mirabegron and vibegron, respectively). Effect sizes at weeks 4 and 12 were calculated using placebo-subtracted

voided versus tolterodine at week 4 , versus mirabegron 25 and $50 \mathrm{mg}$ at week 12 , and versus mirabegron $50 \mathrm{mg}$ at week 52 . For all remaining comparisons, confidence intervals for point estimates for comparisons between vibegron and mirabegron 25 or $50 \mathrm{mg}$ or tolterodine crossed zero, indicating no statistically significant differences among active treatment groups. Both vibegron and mirabegron demonstrated favorable safety profiles across the trials reported. Hypertension was the most frequently occurring AE with mirabegron in the short- and long-term trials, and UTI and hypertension were the most frequently occurring AEs with vibegron in the short- and long-term trials, respectively.

While efficacy data were available for vibegron at week 2 (exploratory endpoint) [12], similar data were not available for mirabegron, and therefore efficacy outcomes at week 4 were analyzed as this was the earliest mutually available time point among all analyzed trials. The indirect comparison at week 4 showed statistically greater improvements for vibegron versus mirabegron 25 and $50 \mathrm{mg}$ for total urinary incontinence episodes. As such, results from this analysis suggest a more rapid onset of changes from baseline; effect size at week 52 was calculated using tolterodine-subtracted changes from baseline. Positive effect sizes favor vibegron; negative effect sizes favor comparators. ER extended release

incontinence reduction with vibegron versus mirabegron 25 or $50 \mathrm{mg}$. Differences in micturitions between vibegron and both doses of mirabegron or tolterodine were not significant at week 4 . Similarly, differences in volume voided at week 4 between vibegron and mirabegron $50 \mathrm{mg}$ were not significant. Results at week 12 were generally similar among all active treatment groups and across all three endpoints, with the exception of significantly greater improvement in volume voided with vibegron compared with both doses of mirabegron.

In these analyses, vibegron but not mirabegron $50 \mathrm{mg}$ showed significant improvement versus tolterodine at week 52 for total incontinence episodes and volume voided, suggesting a potential for more durable efficacy for these endpoints. In the indirect comparison, improvement in total incontinence episodes and volume voided was significantly greater for vibegron compared with mirabegron $50 \mathrm{mg}$ at week 52. All active treatment groups showed similar efficacy with regard to micturitions in the individual trials and in this indirect treatment comparison. Because perception of a lack of efficacy is a common reason for discontinuing anticholinergic medication for $\mathrm{OAB}$ [7], the 
long-term (52-week) efficacy seen with vibegron in randomized controlled trials may translate into improved real-world treatment persistence; however, additional research is needed to fully assess this.

Micturitions and volume voided are standard and generally objective measures used to assess efficacy in OAB clinical trials [27]. Treatment goals include reducing the number of micturitions and increasing volume voided per micturition; however, these measures may not correlate with subjective, patient-reported improvement, and micturitions may be driven by factors outside of pharmacologic manipulation (e.g., fluid intake, voiding out of convenience, rate of bladder filling). Reductions in urgency and incontinence may be associated with improved treatment satisfaction. While significant reductions versus placebo in urgency episodes, when reported, were seen in many of the original trials, definitions for urgency differed among the trials. Therefore, to enable comparison between trials and assess outcomes relevant in real-world settings, total incontinence episodes were incorporated. In this indirect treatment comparison, vibegron showed significantly greater improvement in total incontinence episodes versus placebo and tolterodine across all time points assessed and versus both doses of mirabegron at week 4 and versus mirabegron $50 \mathrm{mg}$ at week 52 .

One potential explanation for differences in efficacy between vibegron and mirabegron may be related to selectivity at $\beta_{3}$-adrenergic receptors. One study suggested an approximately $80 \%$ maximum binding $\left(E_{\max }\right)$ for the $\beta_{3}$ receptor with mirabegron (normalized to isoproterenol) [28]. A separate head-to-head in vitro study also showed an $80 \% E_{\max }$ with mirabegron but a higher, approximately $99 \% E_{\max }$ with vibegron [15].

To the best of our knowledge, this is the first analysis comparing vibegron with mirabegron in patients with $\mathrm{OAB}$. This indirect treatment comparison used rigorous search criteria and statistical methods and is considered an important contributor to the total body of evidence when comparative clinical trials are not available. However, there are limitations inherent to indirect treatment comparisons. The analysis included a single study for vibegron for each time point. Consequently, the overall population receiving vibegron was smaller than the population receiving mirabegron. As a result of differences in trial design, the percentage of patients with incontinence and mean number of incontinence episodes varied at baseline. However, analyses were performed using weighted averages of treatment effects across studies and placebo- or tolterodine-adjusted changes from baseline. Additionally, studies used various definitions for the outcomes assessed (using 3- versus 7-day diaries) and, as noted above, differing classification of urgency and urge urinary incontinence episodes, which may bias or skew results. However, 3- and 7-day diaries have demonstrated similar effectiveness in accurately assessing OAB symptoms [19]. Not all outcomes were assessed at all time points. Reporting of safety outcomes differed between trials, and thus the reporting of AEs may not be comprehensive. This analysis is limited by the assumptions of similarity and consistency among trials, as well as the quality of published methods and results. However, all included studies were high-quality randomized controlled trials, strengthening robustness across all time points.

\section{CONCLUSION}

In this indirect treatment comparison of $\beta_{3^{-}}$ adrenergic receptor agonists for the treatment of $\mathrm{OAB}$ from nine clinical trials, once-daily vibegron $75 \mathrm{mg}$ showed significant improvements at week 4 in total incontinence episodes compared with mirabegron $25 \mathrm{mg}$, mirabegron $50 \mathrm{mg}$, and tolterodine and in volume voided compared with tolterodine. At week 12, vibegron $75 \mathrm{mg}$ showed significant improvements in total incontinence episodes versus tolterodine and in volume voided versus mirabegron 25 and $50 \mathrm{mg}$. Improvements at week 52 with vibegron in total incontinence episodes and volume voided were significantly greater than those with mirabegron $50 \mathrm{mg}$. No significant differences were seen between vibegron and either dose of mirabegron in daily number of micturitions at any time point. The $\beta_{3}$ - 
adrenergic receptor agonists vibegron and mirabegron showed similarly favorable safety profiles. Further, in-depth analyses are needed to evaluate comparative safety, and additional research is needed to determine whether improved efficacy in clinical trials translates into improved treatment persistence in realworld settings.

\section{ACKNOWLEDGEMENTS}

Funding. Funding for this study, for medical writing and editorial support, and for the journal's Open Access Fees was provided by Urovant Sciences.

Authorship. All named authors meet the International Committee of Medical Journal Editors (ICMJE) criteria for authorship for this article, take responsibility for the integrity of the work as a whole, and have given their approval for this version to be published.

Authorship Contributions. All named authors contributed to the conception and design of the present analyses and participated in analysis and interpretation of the data. Statistical analyses were performed by Thomas Rhodes. The first draft of the manuscript was written by medical writers under the direction of all authors, and all authors commented on previous versions of the manuscript and have read and approved the final version.

Medical Writingand Editorial Assistance. Medical writing and editorial support was provided by Krystina Neuman, PhD, CMPP, and Wendy Kandell, PhD, of The Curry Rockefeller Group, LLC (Tarrytown, NY), and was funded by Urovant Sciences (Irvine, CA).

Disclosures. M Kennelly has received grant and/or research study funding from Allergan, Amphora, Astellas, Axonics, Boston Scientific, Coloplast, Cook Myosite, Dignify Therapeutics, EBT Medical, FemPulse, Ipsen, Taris, and Uro1 and is a consultant for Allergan, Astellas, Boston Scientific, Coloplast, Laborie, and Urovant
Sciences. T Rhodes is a consultant for CERobs Consulting, LLC, a consulting firm that was contracted by Urovant Sciences. C Girman is the President of CERobs Consulting, LLC, a consulting firm that was contracted by Urovant Sciences. CERobs Consulting provides consulting services to numerous pharmaceutical companies. E Thomas is an employee of Urovant Sciences. D Shortino and PN Mudd Jr were employees of Urovant Sciences at the time the study was conducted, D Shortino is now an employee of Priovant, Inc and PN Mudd Jr is now an employee of Roivant Sciences.

Compliance with Ethics Guidelines. This article is based on previously published studies where ethics approval and informed consent were obtained. As a result, institutional review board approval and written informed consent were not needed for this analysis.

Data Availability. All data generated or analyzed during this study are included in this published article and its supplementary information files.

Open Access. This article is licensed under a Creative Commons Attribution-NonCommercial 4.0 International License, which permits any non-commercial use, sharing, adaptation, distribution and reproduction in any medium or format, as long as you give appropriate credit to the original author(s) and the source, provide a link to the Creative Commons licence, and indicate if changes were made. The images or other third party material in this article are included in the article's Creative Commons licence, unless indicated otherwise in a credit line to the material. If material is not included in the article's Creative Commons licence and your intended use is not permitted by statutory regulation or exceeds the permitted use, you will need to obtain permission directly from the copyright holder. To view a copy of this licence, visit http://creativecommons.org/licenses/by$\mathrm{nc} / 4.0 /$. 


\section{REFERENCES}

1. Coyne KS, Sexton CC, Vats V, Thompson C, Kopp ZS, Milsom I. National community prevalence of overactive bladder in the United States stratified by sex and age. Urology. 2011;77(5):1081-7.

2. Gormley EA, Lightner DJ, Burgio KL, et al. Diagnosis and treatment of overactive bladder (nonneurogenic) in adults: AUA/SUFU guideline. Linthicum: American Urological Association; 2019.

3. Rai BP, Cody JD, Alhasso A, Stewart L. Anticholinergic drugs versus non-drug active therapies for non-neurogenic overactive bladder syndrome in adults. Cochrane Database Syst Rev. 2012;12(12): CD003193.

4. Szabo SM, Gooch K, Schermer C, et al. Association between cumulative anticholinergic burden and falls and fractures in patients with overactive bladder: US-based retrospective cohort study. BMJ Open. 2019;9(5):e026391.

5. Dmochowski RR, Thai S, Iglay K, et al. Increased risk of incident dementia following use of anticholinergic agents: a systematic literature review and meta-analysis. Neurourol Urodyn. 2021;40(1): 28-37.

6. Song YS, Lee HY, Park JJ, Kim JH. Persistence and adherence of anticholinergics and beta-3 agonist for the treatment of overactive bladder: systematic review and meta-analysis, and network meta-analysis. J Urol. 2021;205(6):1595-604.

7. Benner JS, Nichol MB, Rovner ES, et al. Patient-reported reasons for discontinuing overactive bladder medication. BJU Int. 2010;105(9):1276-82.

8. Kelleher C, Hakimi Z, Zur R, et al. Efficacy and tolerability of mirabegron compared with antimuscarinic monotherapy or combination therapies for overactive bladder: a systematic review and network meta-analysis. Eur Urol. 2018;74(3):324-33.

9. Chapple CR, Nitti VW, Khullar V, et al. Onset of action of the $\beta_{3}$-adrenoceptor agonist, mirabegron, in phase II and III clinical trials in patients with overactive bladder. World J Urol. 2014;32(6): 1565-72.

10. MYRBETRIQ $^{\circledR} \quad$ (mirabegron extended-release tablets). Full prescribing information. Northbrook: Astellas Pharma US, Inc.; 2018.

11. Gratzke C, van Maanen R, Chapple C, et al. Longterm safety and efficacy of mirabegron and solifenacin in combination compared with monotherapy in patients with overactive bladder: a randomised, multicentre phase 3 study (SYNERGY II). Eur Urol. 2018;74(4):501-9.

12. Staskin D, Frankel J, Varano S, Shortino D, Jankowich R, Mudd PN Jr. International phase III, randomized, double-blind, placebo and active controlled study to evaluate the safety and efficacy of vibegron in patients with symptoms of overactive bladder: EMPOWUR. J Urol. 2020;204(2): 316-24.

13. Staskin D, Frankel J, Varano S, Shortino D, Jankowich R, Mudd PN Jr. Once-daily vibegron $75 \mathrm{mg}$ for overactive bladder: long-term safety and efficacy from a double-blind extension study of the international phase 3 trial (EMPOWUR). J Urol. 2021;205(5):1421-9.

14. GEMTESA ${ }^{\circledR}$ (vibegron). Full Prescribing Information. Irvine; 2020.

15. Brucker BM, McHale K, King J, Mudd PN. Selectivity and maximum response of vibegron and mirabegron for $\beta_{3}$-adrenergic receptors. Presented at Society of Urodynamics, Female Pelvic Medicine and Urogenital Reconstruction 2021 Winter Meeting; February 25-27, 2021. Virtual Congress; 2021.

16. Higgins JPT, Thomas J, Chandler J, et al editors. Cochrane handbook for systematic reviews of interventions version 5.1.0. Chichester: Wiley; 2011.

17. Duval S, Tweedie R. A nonparametric "trim and fill" method of accounting for publication bias in metaanalysis. J Am Stat Assoc. 2000;95(449):89-98.

18. Herschorn S, Chapple CR, Abrams P, et al. Efficacy and safety of combinations of mirabegron and solifenacin compared with monotherapy and placebo in patients with overactive bladder (SYNERGY study). BJU Int. 2017;120(4):562-75.

19. Dmochowski RR, Sanders SW, Appell RA, Nitti VW, Davila GW. Bladder-health diaries: an assessment of 3-day vs 7-day entries. BJU Int. 2005;96(7):1049-54.

20. Bucher HC, Guyatt GH, Griffith LE, Walter SD. The results of direct and indirect treatment comparisons in meta-analysis of randomized controlled trials. J Clin Epidemiol. 1997;50(6):683-91.

21. Chapple CR, Kaplan SA, Mitcheson D, et al. Randomized double-blind, active-controlled phase 3 study to assess 12-month safety and efficacy of mirabegron, a $\beta_{3}$-adrenoceptor agonist, in overactive bladder. Eur Urol. 2013;63(2):296-305.

22. Herschorn S, Barkin J, Castro-Diaz D, et al. A phase III, randomized, double-blind, parallel-group, placebo-controlled, multicentre study to assess the efficacy and safety of the $\beta_{3}$ adrenoceptor agonist, 
mirabegron, in patients with symptoms of overactive bladder. Urology. 2013;82(2):313-20.

23. Khullar V, Amarenco G, Angulo JC, et al. Efficacy and tolerability of mirabegron, a $\beta_{3}$-adrenoceptor agonist, in patients with overactive bladder: results from a randomised European-Australian phase 3 trial. Eur Urol. 2013;63(2):283-95.

24. Nitti VW, Auerbach S, Martin N, Calhoun A, Lee M, Herschorn S. Results of a randomized phase III trial of mirabegron in patients with overactive bladder. J Urol. 2013;189(4):1388-95.

25. Yamaguchi O, Marui E, Kakizaki H, et al. Phase III, randomised, double-blind, placebo-controlled study of the $\beta_{3}$-adrenoceptor agonist mirabegron, $50 \mathrm{mg}$ once daily, in Japanese patients with overactive bladder. BJU Int. 2014;113(6):951-60.
26. Kuo HC, Lee KS, Na Y, et al. Results of a randomized, double-blind, parallel-group, placebo- and active-controlled, multicenter study of mirabegron, a $\beta_{3}$-adrenoceptor agonist, in patients with overactive bladder in Asia. Neurourol Urodyn. 2015;34(7): 685-92.

27. Colli E, Parazzini F, Olivieri L, et al. Number of daytime micturitions and volume voided per micturition in the evaluation of efficacy of drugs for overactive bladder: findings from randomized clinical trials. Eur Urol. 2007;52(2):525-30.

28. Takasu T, Ukai M, Sato S, et al. Effect of $(R)-2-(2-$ aminothiazol-4-yl)-4'-\{2-[(2-hydroxy-2-phenylethy1)amino]ethyl\} acetanilide (YM178), a novel selective $\beta_{3}$-adrenoceptor agonist, on bladder function. J Pharmacol Exp Ther. 2007;321(2):642-7. 